\title{
Inferior Vestibular Neuritis
}

\author{
G.M. HALMAGYI, S.T. AW, M. KARLBERG, I.S. CURTHOYS, AND M.J. TODD \\ Neurology Department, Royal Prince Alfred Hospital, Camperdown, \\ NSW 2050, Sydney, Australia
}

\begin{abstract}
Sudden, spontaneous, unilateral loss of vestibular function without simultaneous hearing loss or brain stem signs is generally attributed to a viral infection involving the vestibular nerve and is called acute vestibular neuritis. The clinical hallmarks of acute vestibular neuritis are vertigo, spontaneous nystagmus, and unilateral loss of lateral semicircular function as shown by impulsive and caloric testing. In some patients with vestibular neuritis the process appears to involve only anterior and lateral semicircular function, and these patients are considered to have selective superior vestibular neuritis. Here we report on two patients with acute vertigo, normal lateral semicircular canal function as shown by both impulsive and caloric testing, but selective loss of posterior semicircular canal function as shown by impulsive testing and of saccular function as shown by vestibular evoked myogenic potential testing. We suggest that these patients had selective inferior vestibular neuritis and that contrary to conventional teaching, in a patient with acute spontaneous vertigo, unilateral loss of lateral semicircular canal function is not essential for a diagnosis of acute vestibular neuritis.
\end{abstract}

KEYWORDS: vestibular neuritis; labyrinthitis; vestibulo-ocular reflex; head impulse; canal paresis

\section{INTRODUCTION}

Sudden spontaneous unilateral loss of vestibular function with preserved hearing and no signs of brain stem dysfunction is generally attributed to viral infection and is called acute vestibular neuritis $(\mathrm{aVN}) .^{1,2}$ It has been suggested that reactivation of herpes simplex type 1 virus ${ }^{3}$ could cause aVN in a manner resembling facial palsy (Bell's palsy) and sudden unilateral hearing loss. In patients with aVN, lateral semicircular canal (SCC) function as shown by impulsive or caloric testing is, by definition, abolished or severely reduced on the affected side.

Normally the superior vestibular nerve innervates the SCC, the anterior SCC, and the utricle, whereas the inferior vestibular nerve innervates the posterior SCC and the saccule. In some patients with aVN the process appears to affect predominantly the superior vestibular nerve. In these patients: the axis of the spontaneous nystagmus is aligned with either the lateral SCC alone or the resultant from the lateral and anterior SCCs $;^{4}$ the vestibulo-ocular reflex (VOR) shows asymmetry, suggesting involvement of only the lateral SCC or the lateral and anterior SCCs, sparing the pos-

Address for correspondence: Dr. G.M. Halmagyi, Neurology Department, Royal Prince Alfred Hospital, Missenden Road, Camperdown, NSW 2050, Sydney, Australia. Voice: +61 2515 8300; fax: +61295158347.

michael@icn.usyd.edu.au

Ann. N.Y. Acad. Sci. 956: 306-313 (2002). ( 2002 New York Academy of Sciences. 
terior $\mathrm{SCC} ;{ }^{4}$ about one patient in three with aVN will develop posterior SCC benign paroxysmal positional vertigo, implying preservation of posterior SCC function; ${ }^{5}$ vestibular evoked myogenic potentials (VEMPs) are preserved in two of three patients with aVN, implying preservation of saccular function. ${ }^{6}$

Does aVN sometimes affect only the inferior vestibular nerve and if so what vestibular deficits should such patients have and how would one recognize them? Such patients might have spontaneous nystagmus but it shouldn't be horizontal but should be downbeating and torsional; they will have normal caloric tests since these only measure lateral SCC function. On impulsive testing lateral SCC function should be normal but posterior SCC function should be impaired on one side. The inferior vestibular nerve also innervates the saccule so that VEMPs should also be impaired on same side. Here we report on two patients, each with a single attack of acute spontaneous vertigo, who had just such deficits of vestibular function and suggest that they did indeed have a selective inferior vestibular neuritis.

\section{CASE REPORTS}

CASE 1. The patient, A.T., a 26-year-old IT executive, presented with mild persistent imbalance following a 20 -minute attack of acute spontaneous vertigo with nausea 2 months previously. He had never had headaches or difficulty with hearing, and his health had been unremarkable. On examination there was no spontaneous, gazeevoked, head-shaking, or positional nystagmus and no positional vertigo; the Romberg test on a mat and the Unterberg test both gave negative results.

Results of routine investigations were as follows: electronystagmogram, normal; caloric test: minimal directional preponderance $(30 \%)$ to the left; vestibular evoked myogenic potentials: absent to clicks and taps from the left ear; subjective visual horizontal: 0 degrees (normal); audiogram: normal; MRI brain scan with contrast, normal.

CASE 2. The patient, P.C., a 61-year-old previously well male business executive, developed sudden intense vertigo and nausea while driving home from work. He had to stop his car, vomited, and called for help. He was taken by ambulance to a hospital emergency room. On admission he was in distress with vertigo, retching, and vomiting and was unable to stand. There was no spontaneous or positional nystagmus with or without visual fixation. A CT brain scan gave normal results. He was admitted to the hospital with the provisional diagnosis of cerebellar infarct. The following day he felt better and could stand without support. However, he was unable to hear from his left ear. Over the next 3 days his balance continued to improve but his hearing did not do so.

Routine investigations at that time revealed the following: electronystagmogram: minimal left beating gaze-evoked nystagmus in the dark; caloric tests: normal (R30 $=27$ degrees/s; R44 = 28 degrees/s; L30 = 24 degrees/s; L44 = 20 degrees/s); vestibular evoked myogenic potentials: absent to clicks and to taps from left ear; subjective visual horizontal: 4 degrees to the left (just above our control range of up to 3 degrees): audiogram: severe $(90 \mathrm{~dB})$ flat sensorineural hearing loss left ear; slight conductive loss right ear; MRI brain scan with contrast showed no abnormality, particularly no cerebellar infarction, and no contrast enhancement of the inner ear. 
His balance continued to improve, but 2 weeks later he still rotated to the left on the Unterberger (Fukuda) test and fell on the matted Romberg test. Within 1 month his hearing also improved, but was not back to normal, whereas the VEMPs had returned to normal.

\section{METHODS}

\section{Head Impulses}

\section{The Recording System}

The scleral search coil technique was used to record head and left eye positions using a method previously described. ${ }^{7-10}$ Head and left eye positions were recorded with dual-search coils (Skalar, Delft, The Netherlands) and were precalibrated before each recording. The recording system has a 16-bit resolution and minimum resolution of 0.1 minute of arc with maximum errors and cross-coupling at $2 \%$.

\section{Experimental Protocols}

A head impulse is a passive, unpredictable, low-amplitude (10-20 degree), high acceleration $\left(3,000-4,000\right.$ degrees/ $\left.\mathrm{s}^{2}\right)$ head rotation approximately in the plane of corresponding SCC pairs in yaw, left anterior and right posterior (LARP), or right anterior and left posterior (RALP) directions. ${ }^{10}$ The terms yaw, LARP, and RALP refer to the head impulse directions defined with reference to the subject. Yaw-left, left anterior, and right anterior rotations are positive, whereas yaw-right, right posterior, and left posterior rotations are negative. Counterclockwise direction means that the upper pole of the head or eye is rotated towards the patient's left, and clockwise direction means rotation towards the patient's right.

\section{Data Analysis}

We have expressed three-dimensional head, gaze, and eye positions as rotation vectors. 11 "Head" and "gaze" positions are the orientations in space-fixed coordinates of head and eye, respectively. Eye position is the orientation of eye in headfixed coordinates. Head, gaze, and eye velocities are calculated from their respective positions. Impulsive canal paresis (iCP) is defined as the ratio of gaze velocity to head velocity in SCC coordinates at close to peak head velocity, in response to a head rotation towards the on-direction of a SCC, along its SCC plane. The rationale is that the VOR mediated by SCC receptor hair cells stabilizes gaze during any angular head rotation, but gaze instability only occurs in the absence of SCC function. Impulsive canal paresis measures the gaze instability when the head is rotated towards a paretic SCC. Gaze and head velocities were normalized by dividing each velocity by the magnitude of peak head velocity in each trial. We then determined the gaze and head velocity in SCC coordinates. ${ }^{12,13}$ For each head impulse direction, we determined the mean head and gaze velocity in SCC coordinates from 10 trials. Impulsive canal paresis is determined at $100 \mathrm{~ms}$ from the onset of the head impulse and expressed as a percentage, as follows:

$$
\mathrm{iCP}=\frac{\hat{g}}{\hat{h}} * 100
$$




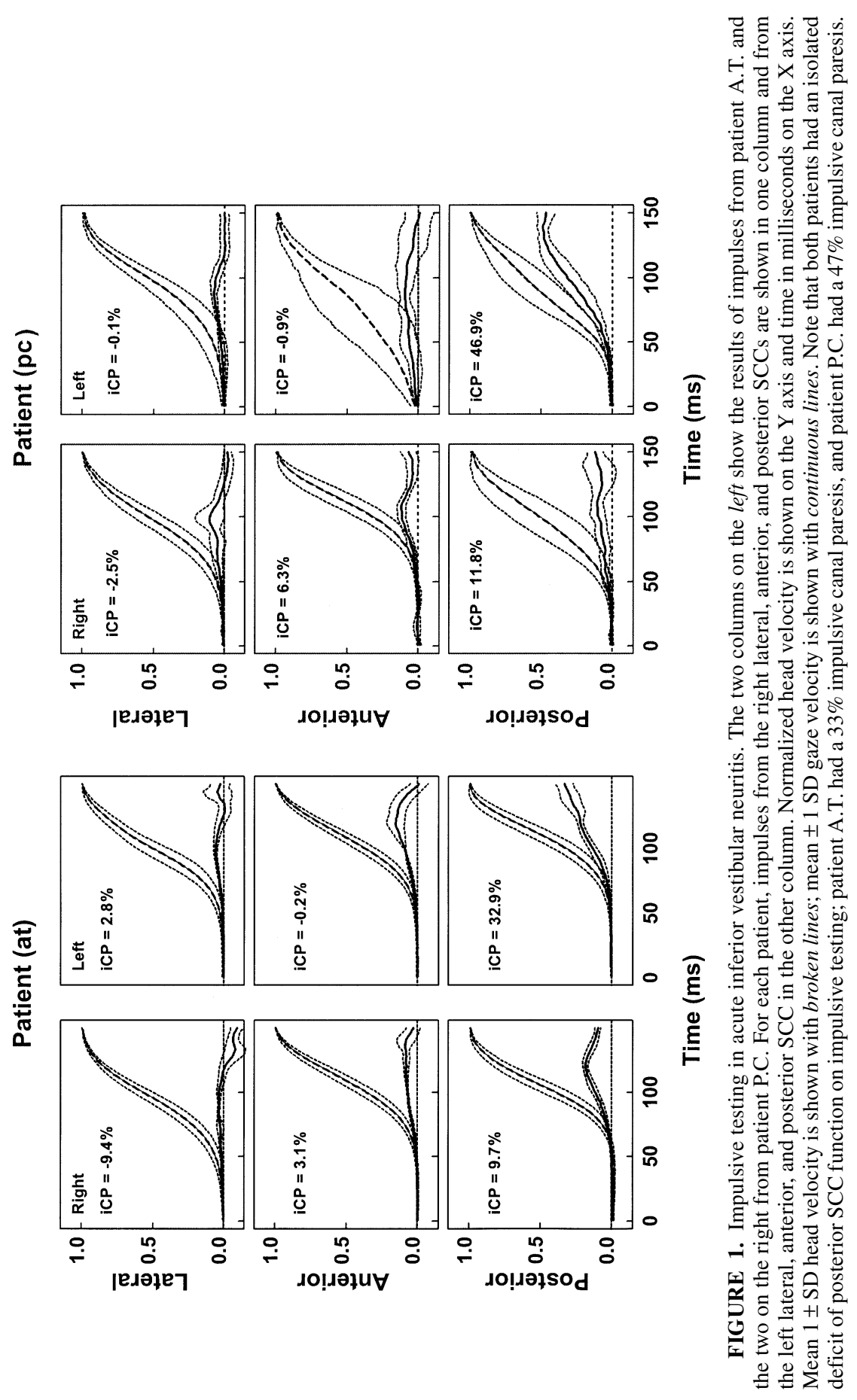




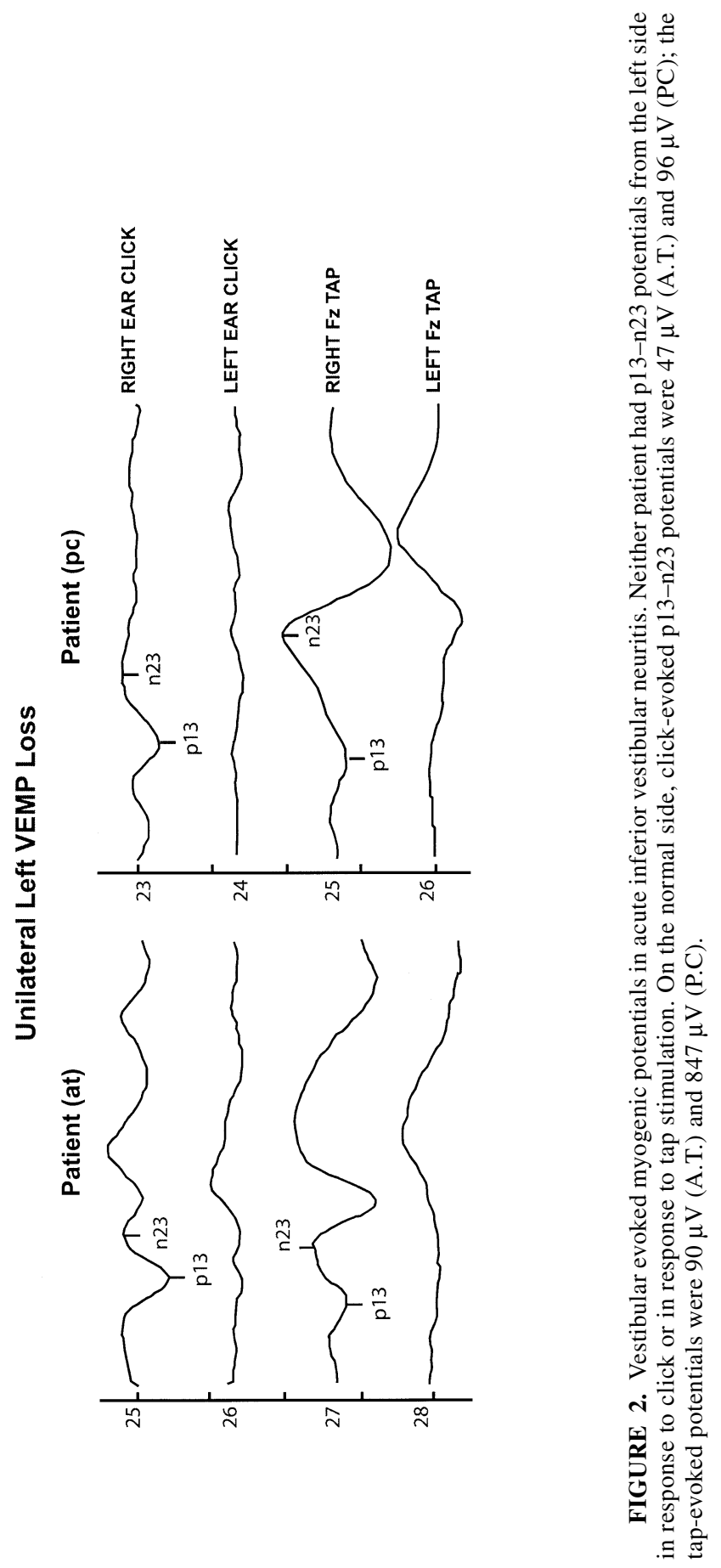


where iCP is the canal paresis, $\hat{h}$ is head velocity in SCC coordinates, and $\hat{g}$ is gaze velocity in SCC coordinates. We excluded the contribution of smooth pursuit by restricting our analysis to an interval of $100 \mathrm{~ms}$ from the onset of the impulse, because smooth pursuit have latencies $\geq 100 \mathrm{~ms}^{14}$

\section{Vestibular Evoked Myogenic Potentials}

Vestibular evoked myogenic potentials were recorded using our standard methodology previously reported. ${ }^{15,16}$ In brief, averaged surface electromyographic potentials were recorded from each sternomastoid muscle, during active contraction of each muscle, in response to loud, brief clicks to each ear and then in response to taps to the forehead. The normal response to such stimuli is a positive potential at around $13 \mathrm{~ms}$ followed by a negative potential at $23 \mathrm{~ms}$, the VEMP. In response to a click the potential appears only in the ipsilateral sternomastoid muscle; in response to a tap the response appears in both sternomastoid muscles. The VEMP is abolished by vestibular nerve section but not in cases of total sensorineural hearing loss; it is progressively attenuated and then abolished by conductive hearing loss. Animal experiments indicate that the VEMP arises from stimulation of the saccule. ${ }^{17-20}$

\section{RESULTS}

FIGURE 1 shows the results of impulsive testing. Head velocities stimulating the affected posterior SCC produce a significant gaze perturbation, so that the value of iCP is $33 \%$ in patient 1 and $47 \%$ in patient 2 (normal $12 \pm 7 \%$ ). By contrast, head velocities stimulating the anterior and lateral SCCs on the affected side and in the planes of all three SCCs on the normal side produced only slight perturbutations of gaze, so that the values for iCP were within or just above the control range (lateral $\mathrm{SCC}=0 \pm 5 \%$; anterior $7 \pm 7 \%$; posterior $12 \pm 7 \%) .{ }^{21}$ FIGURE 2 shows that VEMPs were absent from the affected side in both patients, both in response to clicks and in response to taps.

\section{DISCUSSION}

Three-dimensional measurement and vector analysis of the angular VOR in response to the head impulse test are an established method of evaluating individual SCC function. ${ }^{8-10,21,22}$ The SCC planes do not lie along traditional head planes, yaw (i.e., horizontal), pitch (i.e., sagittal), and roll (i.e., coronal or frontal). For example, the plane of lateral SCC is oriented about $25^{\circ}$ above the yaw plane, as defined by Reid's line. ${ }^{12}$ Interpretation of impulsive tests requires familiarity with the reference planes and directions for a three-dimensional description of the VOR. Therefore, VOR gain and canal paresis are best understood when expressed in SCC planes.

We define iCP as the ratio of gaze velocity to head velocity in SCC planes at close to peak head velocity, and use iCP to quantify individual SCC deficits. The VOR mediated by a normal SCC stabilizes gaze when the head is rotated towards the SCC, so that gaze velocity would be close to zero. ${ }^{8}$ Our data show that iCP from a normal SCC is $\leq 12 \%$. When the head is rotated towards an abnormal SCC, gaze is unstable, 
so that gaze velocity is high. ${ }^{9,10}$ Mean iCP values from an affected SCC after aVN and after surgical deafferentation is $\geq 65 \%$.

The iCP values from normal vertical SCCs are slightly higher due to a lower $(0.7)$ torsional VOR gain in response to head impulses in the roll plane even in normal subjects. ${ }^{8}$ As the vertical SCCs are positioned approximately halfway between the pitch and roll planes, a smaller roll-torsional gain will result in the higher $\mathrm{iCP}$ value when gaze velocity is resolved into SCC planes.

A head impulse stimulates both the on- and off-directions of a pair of SCCs, that is, left and right lateral SCCs (yaw plane), right anterior and left posterior SCCs (RALP plane), and left anterior and right posterior SCCs (LARP plane). The iCP value from in-plane SCC on the side opposite an abnormal SCC would be slightly higher because of the "push-pull" behavior of the SCCs; the contribution from disinhibition is absent, ${ }^{23}$ therefore, the iCP value is higher than from normal subjects.

The two patients we report on here both presented with acute spontaneous vertigo and had little or no spontaneous nystagmus, normal lateral SCC function but unilateral loss of posterior SCC function and saccular function, features that we propose are the hallmarks in inferior vestibular neuritis.

The likely reason that patients with inferior vestibular neuritis are difficult to recognize clinically is that with conventional testing they have little spontaneous nystagmus during the acute phase of the illness and have normal caloric responses. Without these clinical signs, a patient with inferior vestibular neuritis would not fulfill the conventional clinical criteria of aVN and therefore would be considered to have a central vestibular lesion. This is particularly so, because it has been reported that one in five patients with acute pontine lesions present with signs that mimic acute unilateral peripheral vestibulopathy. ${ }^{24}$ Therefore, until now, patients with inferior vestibular neuritis would have needed some other clinical feature to indicate that the cause of the acute vertigo was acute unilateral peripheral vestibulopathy. In one of our cases, that additional feature was simultaneous ipsilateral hearing loss. Our patients had normal lateral SCC function on caloric and impulsive testing but a posterior SCC deficit on impulsive testing, indicating that they had selective involvement of the inferior vestibular nerve; however, it was simultaneous ipsilateral hearing loss in one patient that indicated to the attending clinicians that a unilateral inner ear disturbance was the likely cause of the problem.

\section{ACKNOWLEDGMENTS}

This work was supported by the Garnett Passe and Rodney Williams Memorial Foundation, the Australian National Health and Medical Research Council, the Swedish Medical Research Council, the Wenner-Gren Foundation, The Maggie Stephens Foundation, and the Neurology Department Trustees, Royal Prince Alfred Hospital.

\section{REFERENCES}

1. Dix, M.R. \& C.S. HallPike. 1952. The pathology, symptomatology and diagnosis of certain common disorders of the vestibular system. Ann. Otol. Rhinol. Laryngol. 61: 987-1016. 
2. StRupp, M. \& T. BRAndT. 1999. Vestibular neuritis. In Vestibular Dysfunction and Its Therapy. U. Buttner, ed. Adv. Otorhinolaryngol. 55: 111-136.

3. Schulz, P., V. Arbusow, M. Strupp, et al. 1998. Highly variable distribution of HSV1-specific DNA in human geniculate, vestibular and spiral ganglion. Neurosci. Lett. 252: $139-142$.

4. Fetter, M. \& J. Dichgans. 1996. Vestibular neuritis spares the inferior division of the vestibular nerve. Brain 119: 755-763.

5. BÜCHELE, W. \& T. BRANDT. 1988. Vestibular neuritis: a horizontal semicircular canal paresis? Adv. Otorhinolaryngol. 42: 157-161.

6. Murofushi, T., G.M. Halmagyi, R.A. Yavor \& J.G. Colebatch. 1996. Absent vestibular evoked myogenic potentials in vestibular neurolabyrinthitis. Arch. Otolaryngol. Head Neck Surg. 122: 845-848.

7. Robinson, D.A. 1963. A method of measuring eye movement using a scleral search coil in a magnetic field. IEEE Trans. Biomed. Eng. 10: 137-145.

8. Aw, S.T., T. Haslwanter, G.M. Halmagyi, et al. 1996. Three-dimensional vector analysis of the human vestibuloocular reflex in response to high-acceleration head rotations. I. Responses in normal subjects. J. Neurophysiol. 76: 4009-4020.

9. Aw, S.T., G.M. Halmagyi, T. HaSLwANTER, et al. 1996. Three-dimensional vector analysis of the human vestibuloocular reflex in response to high-acceleration head rotations. II. Responses in subjects with unilateral vestibular loss and selective semicircular canal occlusion. J. Neurophysiol. 76: 4021-4030.

10. Cremer, P.D., G.M. Halmagyi, S.T. Aw, et al. 1998. Semircircular canal plane head impulses detect absent function of individual semicircular canals. Brain 121: 699716.

11. Haustein, W. 1989. Considerations on Listing's law and the primary position by means of a matrix description of eye position control. Biol. Cybern. 60: 411-420.

12. Blanks, R.H.I., I.S. CuRThoys \& C.H. Markham. 1975. Planar relationships of the semicircular canals in man. Acta Otolaryngol. 80: 185-196.

13. Aw, S.T., T. Haslwanter, M. Fetter, et al. 1998. Contribution of the vertical semicircular canals to the caloric nystagmus. Acta Otolaryngol. 118: 618-627.

14. Carl, J.R. \& R.S. Gellman. 1987. Human smooth pursuit: stimulus-dependent responses. J. Neurophysiol. 57: 1446-1463.

15. Colebatch, J.G., G.M. Halmagyi \& N.F. Skuse. 1994. Myogenic potentials generated by a click-evoked vestibulocollic reflex. J. Neurol. Neurosurg. Psychiatry 57: 190-197.

16. Halmagyi, G.M., R.A. Yavor \& J.G. Colebatch. 1995. Tapping the head activates the vestibular system: a new use for the clinical reflex hammer. Neurology 45: 19271929.

17. Murofushi, T. \& I.S. Curthoys. 1997. Physiological and anatomical study of clicksensitive primary vestibular afferents in the guinea-pig. Acta Otolaryngol. (Stockh.) 117: $66-72$.

18. Murofushi, T., I.S. Curthoys \& D.P. Gilchrist. 1996. Response of guinea pig vestibular nucleus neurons to clicks. Exp. Brain Res. 111: 149-152.

19. Murofushi, T., I.S. Curthoys, A.N. Topple, et al. 1995. Responses of guinea pig primary vestibular neurons to clicks. Exp. Brain Res. 103: 174-178.

20. Kushiro, K., M. ZaKir, Y. OGawa, et al. 1999. Saccular and utricular inputs to sternocleidomastoid motoneurons of decerebrate cat. Exp. Brain Res. 126: 410-416.

21. Aw, S.T., M. Fetter, P.D. Cremer, et al. 2001. Individual semicircular function in superior and inferior vestibular neuritis. Neurology 57: 768-774.

22. Schmid-Priscoveanu, A., D. Straumann, A. Bohmer \& H. Obzina. 1999. Vestibuloocular responses during static head roll and three-dimensional head impulses after vestibular neuritis. Acta Otolaryngol. 119: 750-757.

23. Shimazu, H. \& W. Precht. 1966. Inhibition of central vestibular neurons from the contra-lateral labyrinth and its mediating pathway. J. Neurophysiol. 29: 467-492.

24. Thomke, F. \& H.C. Hopf. 1999. Pontine lesions mimicking acute peripheral vestibulopathy. J. Neurol. Neurosurg. Psychiatry 66: 340-349. 